\title{
Combining lung ultrasound and wells score for diagnosing pulmonary embolism in critically ill COVID-19 patients
}

Viviane Zotzmann ( $\nabla$ viviane.zotzmann@universitaets-herzzentrum.de )

Universitats-Herzzentrum Freiburg https://orcid.org/0000-0003-1335-4732

Corinna Nadine Lang

Universitats-Herzzentrum Freiburg

Tobias Wengenmayer

Universitats-Herzzentrum Freiburg

Xavier Bemtgen

Universitats-Herzzentrum Freiburg

Bonaventura Schmid

Universitats-Herzzentrum Freiburg

Katharina Mueller-Peltzer

Diagnostic and Interventional Radiology, University of Freiburg

Alexander Supady

Universitats-Herzzentrum Freiburg

Christoph Bode

Universitats-Herzzentrum Freiburg

Daniel Duerschmied

Universitats-Herzzentrum Freiburg

Dawid L Staudacher

Universitats-Herzzentrum Freiburg

Research

Keywords: COVID-19, Lung ultrasound, CTPA, pulmonary embolism, SARS-CoV2, Wells score,

Posted Date: September 8th, 2020

DOl: https://doi.org/10.21203/rs.3.rs-70085/v1

License: (c) (1) This work is licensed under a Creative Commons Attribution 4.0 International License.

Read Full License 


\section{Abstract}

Introduction: Subpleural consolidations have been found in lung ultrasound in patients with COVID-19, possibly deriving from pulmonary embolism (PE). The diagnostic utility of impact of lung ultrasound in critical-ill patients with COVID-19 for PE diagnostics however is unclear.

Methods: We retrospectively evaluated all SARS-CoV2-associated ARDS patients admitted to our ICU between March $8^{\text {th }}$ and May $31^{\text {th }} 2020$. They were enrolled in this study, when a lung ultrasound and a computed tomography pulmonary angiography (CTPA) were documented. In addition, wells score was calculated to estimate the probability of PE. The CTPA was used as the gold standard for the detection of PE.

Results: Twenty patients met the inclusion criteria. In 12/20 patients (60\%) (sub-) segmental PE were detected by CT-angiography. Lung ultrasound found subpleural consolidations in $90 \%$ of patients. PEtypical large supleural consolidations with a size $\geq 1 \mathrm{~cm}$ were detectable in $65 \%$ of patients and were significant more frequent in patients with PE compared to those without $(p=0.035)$. Large consolidations predicted PE with a sensitivity of $77 \%$ and a specificity of $71 \%$. The Wells score was significantly higher in patients with PE compared to those without $(2.7 \pm 0.8$ and $1.7 \pm 0.5$, respectively, $p=0.042)$ and predicted $P E$ with an AUC of 0.81 .

When combining the two modalities, comparing patients with considered/probable PE using LUS plus a Wells score $\geq 2$ to patients with possible/unlikely PE in LUS plus a Wells score $<2$, PE could be predicted with a sensitivity of $100 \%$ and a specificity of $80 \%$.

Conclusion: Large consolidations detected in lung ultrasound were found frequently in COVID-19 ARDS patients with pulmonary embolism. In combination with a Wells score $>2$, this might indicate a high-risk for PE in COVID-19.

\section{Introduction}

In hospitalized patients infected with the severe acute respiratory syndrome coronavirus 2 (SARS-CoV-2), respiratory failure is a common complication. Several case reports and retrospective registry analyses report a high rate of thrombotic complications including pulmonary embolism (PE) and microvascular thrombosis [1-3]. In a recent analysis, patients with severe coronavirus disease 2019 (COVID-19) who were treated with low-molecular weight heparin $(\mathrm{LMWH})$ had a lower 28-day mortality compared with similarly-ill patients that were not treated with LMWH [4].

Since the beginning of the outbreak multiple studies evaluating imaging techniques described bilateral ground-glass opacities, crazy-paving and air-space consolidations in peripheral and basal distribution in patients with COVID-19 pneumonia [5, 6]. Zieleskiewicz et al. were able to show that the assessment of the LUS score was an indication of the severity of pneumonia, which was evaluated by a chest CT scan [7]. 
Peng et al and Volpicelli et al. described COVID-19-typical signs in lung ultrasound (LUS). These signs can already be useful in the emergency department for risk stratification and for underpinning the diagnostic assurance [8-10].

Subpleural consolidations are among those COVID-19-specific sonographic signs, described by Peng et.al [8]. As elegantly described earlier by Reissig et al., these consolidations are well known and described sonographic criteria for peripheral or segmental PE with high sensitivity and specificity $(76.9 \%$ and $91.3 \%$, respectively). Therefore, it might be possible that these COVID-19-typical ultrasound-signs not only mimic but rather actually picture a peripheral or (sub-)segmental PE [11].

In order to address this issue of immediate therapeutic relevance, we conducted a retrospective registry analyses to investigate if lung ultrasound in critical-ill COVID-19-patients could help to diagnose PE manifestations.

\section{Methods}

This is an investigator-initiated retrospective non-interventional registry study. All data were collected retrospectively from patient records at the University of Freiburg Medical Center.

\section{Study Population And Data Collection}

We recruited all patients reverse transcriptase polymerase chain reaction (rtPCR)-confirmed SARS-CoV-2 infection treated at the medical intensive care unit (ICU) at the University of Freiburg Medical Center between March 8th and May 31th, 2020 if they fulfilled all of the following inclusion criteria:

1. respiratory failure as defined by ARDS with a Horowitz index less than $100 \mathrm{mmHg}$ due to SARS-CoV2

2. lung ultrasound and

3. contrast enhanced CT-scan with pulmonary angiography (CTPA) were performed and documented.

All data for this study was taken from the electronic patients files. As data were collected retrospectively, no interventions were applied for the purpose of this study and all patients were treated according to current treatment standards and guidelines.

\section{Diagnostic Pathway During ICU Course}

Our ICU is located at a university hospital offering a 24/7 ECMO center specialized in acute respiratory distress syndrome (ARDS). ARDS treatment is performed according to current guidelines, including early mobilization or prone positioning and early spontaneous breathing in patients without desynchronization with the ventilator[12]. In case of severe pulmonary failure, a multidisciplinary team including at least the intensivist in charge, an ECMO specialist, a registered nurse and a perfusionist decide about extracorporeal membrane oxygenation (ECMO) in severe courses where invasive mechanical ventilation is not sufficient. During the SARS-CoV2 pandemic, daily LUS was encouraged by local standard operating 
procedures when deterioration in respiratory function was evident .Imaging (sonography, including LUS, echocardiography and sonography) was performed by experienced intensivists. A CTPA was performed when indicated by the intensivist and radiologist in charge.

\section{Lung Ultrasound (LUS)}

Experienced intensivists with advanced knowledge in sonography carried out the LUS. Evaluation and assessment of the lung sonography findings was mostly done with knowledge of the d-dimer value, but before performing a CTPA and therefore prospectively blinded with regard to the CTPA result. LUS examination was carried out using a Philips CX50 echocardiography machine with a multifrequency probe C5-1 (5 - $1 \mathrm{MHz})$ or L12-3 (12-3 MHz). Alternatively, a Philips Sparq with a multifrequency probe C6-2 (6 - $2 \mathrm{MHz})$ or L12-4 (12-4 MHz) was used. Due to our local standard, we used an adjusted BLUE protocol for investigating the lungs. The BLUE protocol [13] is a standardized diagram for the rapid identification of $97 \%$ of the causes of dyspnea in adult patients (pulmonary edema, pneumonia, $\mathrm{PE}$, COPD, asthma, pneumothorax). Ultrasound examinations were performed along the midclavicular line in the bilateral anterior chest wall and the scapular line and interscapular regions in the posterior chest wall - each right and left side of the chest - at the bedside. Since the mechanically ventilated intensive care patients can usually only be examined either lying on the front or back, examination of all 12 lung fields was only possible in individual cases, mostly only 8 fields could be examined reliably. Furthermore, we focused on the COVID-19-typical signs as described before [8], such as multiple B-lines (comet-tail artefacts) in a variety of patterns (focal, multifocal and confluent), a thickening of the pleural line with irregularity and consolidations in a variety of patterns (see Fig. 2). On a LUS survey sheet we documented the number of fields examined as well as the patterns described above (B-Lines, consolidations, pleurairregularities) for each individual field. Finally, the summary of the examination assessed whether it was suitable for COVID-19 and whether PE was highly likely, probable, possible or unlikely.

The diagnosis of PE suggested by Mathis et al., based on the number and size of the subpleural consolidations, was used but slightly modified. Mathis et al. describe consolidations with a size of more than $5 \mathrm{~mm}$ as typical for PE[14]. Due to the pronounced pleural changes in COVID-19 with a significant thickening of the pleura, we only considered triangular consolidations $\geq 1 \mathrm{~cm}$ as PE-typical, $<1 \mathrm{~cm}$ as non-typical for PE. The following criteria for detection of PE diagnosis were used:

$P E$ is considered highly likely when two or more characteristic triangular lesions ( $\geq 1 \mathrm{~cm})$ were demonstrated; $P E$ is considered probable: if one characteristic triangular lesion ( $\geq 1 \mathrm{~cm})$ was detected; $P E$ is considered possible: if two (or more) non-typical lesions $(<1 \mathrm{~cm}$ ) were detected; PE is considered unlikely: neither typical nor atypical consolidations.

To assess hemodynamic relevance of the PE, additionally, right heart echocardiography and sonography of the vena cava were performed.

\section{CTPA Examination Protocol And Imaging Analysis}


CTPA scans were performed using a commercial CT scanner (SOMATOM Definition Flash; Siemens Healthineers $\mathrm{GmbH}$, Forchheim, Germany) with the following scanning parameters: tube voltage, $100 \mathrm{kV}$; tube current, $90 \mathrm{mAs}$; rotation time, $0.28 \mathrm{~s}$. $128 \times 0.6 \mathrm{~mm}$ collimation with automated dose modulation (CARE dose4D, Siemens Healthineers GmbH, Forchheim, Germany). Patients without extracorporeal membrane oxygenation (ECMO) received the standard pulmonary angiography protocol with bolustracking method of $70 \mathrm{ml}$ contrast agent (Imeron 400, Bracco Imaging, Germany). To consider an altered blood flow in patients with ECMO device the amount of contrast agent was adjusted to $100 \mathrm{ml}$ and the ROI was placed in the air. The scan was manually started when an adequate contrast was visually detected in the pulmonary trunk. If tolerated by the patient ECMO flow was reduced to 70 to $50 \%$ of the initial value after scout acquisition for the time of the contrast-enhanced scan. Each pulmonary lung segment was separately evaluated for parenchymal abnormalities (ground-glass opacities and/or crazypaving pattern, and air space consolidation) and PE.

A segmental or subsegmental PE was defined as central filling defect within a vessel surrounded by contrast material when orthogonal or parallel to the long axis of the vessel as well as eccentric filling defect rendering an acute angle with the vessel wall as well as complete occlusion of a dilated vessel [15].

\section{Wells Score}

The Wells score is a well-established screening tool for PE and is used in everyday care to assess the clinical pre-Test probability at our institution [16]. The following questions are scored: Clinical signs and symptoms of deep vein thrombosis (DVT)? (+3), PE is \#1 diagnosis or equally likely? (+3), heart rate > 100 bpm? (+1.5), Immobilization at least 3 days OR surgery in the previous 4 weeks? (+ 1.5), previous objectively diagnosed PE or DVT? (+ 1.5), hemoptysis? (+ 1) and malignancy with treatment within 6 months or palliative? $(+1)$. The largest study [16] using a three-tier Model and demonstrated risk stratification with: a.) Low score of 1-2 points having a 1.3\% prevalence, b.) Moderate score of 2-6 points having a $16.2 \%$ prevalence or c.) High score of > 6 points having a $37.5 \%$ prevalence. In order to reduce inter-observer variance for our research, the Wells score was retrospectively re-assessed for the day of CTPA by a single intensivist considering all available data.

\section{Statistical Analysis And Ethics}

Statistical analyses were performed using SPSS, version 23.0, (IBM, New York City, USA). Graphs were drawn with Prism, version 8.4.3 (GraphPad, San Diego, USA).

Continuous variables were compared using student's T-Test, Fisher's exact test was used for contingency table analyses. Significance level was set at $p<0.05$. The study confirms with the 1975 declaration of Helsinki and it was approved by the ethics committee of the Albert Ludwig University of Freiburg (file number $234-20)$.

\section{Results}




\section{Patient selection and characteristics}

A total of 113 patients were admitted to the ICU within the observed time period, of these 25 patients were diagnosed with severe respiratory failure due to COVID-19. Four Patients had to be excluded from the study because they did not receive a CTPA, in one patient LUS could not be reliably evaluated due to huge parenchymal pulmonary bleeding and hemothorax. Therefore, 20 patients could be enrolled in this study (see Fig. 1). Mean age (+/- S.D.) of the patients was $61.6 \pm 9.9$ years, SAPS II score was $48.4 \pm 12.4$ on day 1 after admission to ICU. Eighteen of twenty patients (90\%) were intubated. Eleven from $20(55 \%)$ patients were on ECMO during their ICU stay. For more detailed patient characteristics see Table 1.

Table 1

Patients characteristics of all patients, with ARDS due to COVID-19, which underwent LUS, Wells score and CTPA: The number within the two groups (PE vs. non-PE) as well as the percentage in relation to the entire group or the standard deviation are given.

\begin{tabular}{|c|c|c|c|}
\hline Characteristics & with PE & no PE & $\mathrm{p}$ value $<0.05^{*}$ \\
\hline number & $12.0(60 \%)$ & $8.0(40 \%)$ & 0.690 \\
\hline age & $59.0 \pm 8.0$ & $65.5 \pm 11.8$ & 0.190 \\
\hline female & $4.0(20.0 \%)$ & $2.0(10.0 \%)$ & 0.690 \\
\hline $\mathrm{BMI}[\mathrm{kg} / \mathrm{m} \square]$ & $26.9 \pm 3.6$ & $30.4 \pm 8.8$ & 0.285 \\
\hline ICU-mortality & $5.0(25 \%)$ & $4.0(20 \%)$ & 0.713 \\
\hline ICU-stay [in days] & $27.3 \pm 26.8$ & $30.0 \pm 23.3$ & 0.819 \\
\hline TISS 10 - Score & $17.0 \pm 6.7$ & $15.4 \pm 5.8$ & 0.500 \\
\hline SAPS 2 - Score & $50.0 \pm 10.4$ & $45.9 \pm 15.4$ & 0.395 \\
\hline d-dimers $[\mathrm{mg} / \mathrm{l}]$ (at time of LUS) & $16.3 \pm 13.3$ & $13.5 \pm 12.6$ & 0.579 \\
\hline d-dimers $[\mathrm{mg} / \mathrm{l}]$ (at time of admission) & $6.7 \pm 6.0$ & $4.2 \pm 3.0$ & 0.526 \\
\hline wells score (at time of LUS) & $2.7 \pm 0.8$ & $1.7 \pm 0.5$ & 0.042 \\
\hline therapeutic anticoagulation (at time of admission) & $1.0(5.0 \%)$ & $4.0(20.0 \%)$ & 0.035 \\
\hline Echocardiography: PAP sys. [mmHg] & $46.8 \pm 18.9$ & $42.6 \pm 16.0$ & 0.563 \\
\hline invasive mechanical respiratory support [in days] & $28.8 \pm 29.4$ & $29.1 \pm 25.0$ & 0.988 \\
\hline on ECMO support & $7(35 \%)$ & $4(20 \%)$ & 0.713 \\
\hline \multicolumn{4}{|l|}{ pre-existing co-morbidities: } \\
\hline Lung disorder & $1.0(5.0 \%)$ & $4.0(20.0 \%)$ & 0.035 \\
\hline Tobacco smoke & $3.0(15.0 \%)$ & $5.0(25.0 \%)$ & 0.094 \\
\hline Diabetes mellitus & $3.0(15.0 \%)$ & $0.0(0.0 \%)$ & 0.125 \\
\hline Arterial hypertension & $3.0(15.0 \%)$ & $4.0(20.0 \%)$ & 0.251 \\
\hline Heart failure & $2.0(10.0 \%)$ & $3.0(15.0 \%)$ & 0.292 \\
\hline Kidney failure & $1.0(0.5 \%)$ & $1.0(5.0 \%)$ & 0.761 \\
\hline Liver failure & $0.0(0.0 \%)$ & $1.0(5.0 \%)$ & 0.210 \\
\hline Coagulopathy & $1.0(5.0 \%)$ & $0.0(0.0 \%)$ & 0.402 \\
\hline Immunodeficiency & $3.0(15.0 \%)$ & $0.0(0.0 \%)$ & 0.125 \\
\hline Obesity (BMI > 30) & $2.0(10.0 \%)$ & $2.0(10.0 \%)$ & 0.648 \\
\hline
\end{tabular}




\section{Computed Tomography Pulmonary Angiography (CTPA)}

The final diagnosis of PE was confirmed by CTPA. In total 300 lung segments were evaluated. In 12 out of 20 patients (60\%), segmental and subsegmental PE were detected. In patients with PE 62 segments in total with an average of $2.90 \pm 3.38$ lung segments were affected (range 1 to 9 ). The analysis of distribution of PE on lung lobe level, showed the right lower lobe to be affected in 10 out of 12 patients with $\mathrm{PE}$, followed by the right upper lobe which was affected in 8 out of 12 patients. The complete PE distribution of the entire lung is shown in Fig. 1 of the supplement.

\section{LUS In Covid-19}

LUS examinations showed abnormal lung ultrasound findings, with pleural abnormalities including thickening of the pleural line with pleural line irregularity in nineteen of twenty cases (95\%). B-lines, in a variety of patterns including focal, multifocal, and confluent could also be documented in 19 from 20 (95\%) patients. Multifocal B-lines were found in 14 patients and confluent in 11 patients, however B-line pattern was heterogeneous in individual patients. Subpleural consolidations were found in 18/20 (90\%) patients. Typical consolidations with a size / depth of $>1 \mathrm{~cm}$ were detectable in 13/20 (65\%) patients. Ten $(50 \%)$ patients showed atypical consolidations $(<1 \mathrm{~cm})$. Typical and atypical consolidations could occur co-dominantly in the same individual. The COVID-19-typical lung sonographic findings are summarized in Table 2. Sonographic image-examples of these different pleural morphologies are shown in Fig. 2.

\begin{tabular}{|ll|}
\hline \multicolumn{2}{|c|}{ Table 2 } \\
$\begin{array}{c}\text { COVID-typical Lung ultrasound findings } \\
\text { of the 20 patients included. Data are } \\
\text { presented as percentage (number of } \\
\text { cases with findings) }\end{array}$ \\
\hline Pleural line abnormalities & $95 \%(19)$ \\
\hline B-lines & $95 \%(19)$ \\
\hline - multifocal B-lines & $70 \%(14)$ \\
\hline - confluent B-lines & $55 \%(11)$ \\
\hline Supleural Consolidations & $90 \%(18)$ \\
\hline - Typical $(\geq 1 \mathrm{~cm})$ & $65 \%(13)$ \\
\hline - Atypical $(<1 \mathrm{~cm})$ & $50 \%(10)$ \\
\hline Pleural effusion & $10 \%(2)$ \\
\hline Pericardial effusion & $5 \%(1)$ \\
\hline
\end{tabular}

\section{Wells Score}

Average Wells score in the entire cohort was $2.3 \pm 0.8$. In the group with evidence of $P E$, the wells score was significantly higher than in the group without $P E(2.7 \pm 0.8$ in patients with respectively $1.7 \pm 0.5$ in patients without $P E, p=0.042$ ). 
Using LUS and the criteria described above and suggested by [14], PE was considered in 12/20 (60\%) patients, probable in $1 / 20(5 \%)$, possible in $2 / 20(10 \%)$, and unlikely in $5 / 20(25 \%)$ patients. When forming a two-tier scale of probable PE (considered/probable PE in LUS versus possible/unlikely PE in LUS), pulmonary embolism could be predicted with an AUC of 0.729 and a sensitivity of $77 \%$ and a specificity of $71 \%$, see Fig. 2. The Wells score estimated the risk for PE as very high (Wells score $>6$ ) in $0 / 20$ patients, as moderate (score $2-6$ ) in $10 / 20$ patients and as low (score $<2$ ) in 10/20 patients. With the Wells score, PE could be predicted with an AUC of 0.813 and a sensitivity of $90 \%$ and a specificity of $70 \%$, see Fig. 2. When combining the two modalities, comparing patients with considered/probable PE using LUS plus a Wells score $\geq 2$ to patients with possible/unlikely PE using LUS plus a Wells score $<2$, PE could be predicted with an AUC of 0.944 and a sensitivity of $100 \%$ and a specificity of $80 \%$, see Fig. 3 .

\section{Discussion}

Using a standardized LUS exam, focusing on subpleural consolidations $\geq 1 \mathrm{~cm}$, predicted PE in patients with COVID-19 ARDS with a specificity of $83.3 \%$ and a sensitivity of $77 \%$.

Subpleural consolidations have been described as typical signs for COVID-19. In addition, these consolidations have been described as sonographic criteria for peripheral or segmental PE with high sensitivity and specificity (76.9\% and $91.3 \%$, respectively) [14] in non-COVID-19 patients. So far, however, LUS for detection of PE has not been validated in COVID-19. Our results might confirm the findings that were generally obtained for peripheral PE in patients without COVID-19 [14, 19, 20]. For the sonographic assessment of the PE-probability, we followed the classification suggested elegantly by Mathis et al. whoever using a two-tier scale as described above.

Lung sonography has developed considerably in recent years and is therefore widely used with regard to its clinical application. Although the method is dependent on the examiner and requires a certain amount of practice, it is easy to learn, available everywhere, can be carried out without radiation exposure or the use of contrast agent and is cost-effective compared to methods such as CT-examinations or ventilation perfusion scintigraphy. In addition, infectious patients do not have to be transported as ultrasound can be performed on the bed side (point-of-care). Since LUS can identify changes in superficial lung tissue that correlate with histopathological findings which could only be identified in CT-scan, but remain hidden in a large percentage of chest X-rays, the role of LUS in the context of viral pneumonia may be relevant [17]. In view of the recent outbreak of pneumonia from Wuhan, China, in December 2019 by the SARS-CoV2 pathogen, which is referred to as COVID-19, lung sonography proved to be useful $[7,17,18]$. Typical signs for COVID-19 have been defined, which might be particularly helpful in the emergency rooms for diagnosis and triage $[17,18]$. Our study confirms the signs described by Peng et al. in all but one COVID19 patient included in the study thou consolidations are not exclusive for COVID-19.

However, with the LUS alone we might overestimate the occurrence of PE (false-positive 3/8 patients, $37.5 \%$ ). When increasing the threshold to 2 subpleural consolidations $\geq 1 \mathrm{~cm}$ for diagnosis for $\mathrm{PE}$, sensitivity drops. In order to improve specificity, a combination with a second method could be useful. 
The Wells score has also not yet been validated in patients with COVID-19. Although the results of the Wells score were very homogeneous in our cohort, we could predict PE with the Wells score alone with an AUC of 0.813 , a sensitivity of $90 \%$ and a specificity of $70 \%$. When combining the two modalities, comparing patients with considered/probable PE using LUS plus a Wells score $\geq 2$ to patients with possible/unlikely PE using LUS plus a Wells score $<2$, pulmonary embolism could be predicted with an AUC of 0.944 and a sensitivity of $100 \%$ and a specificity of $80 \%$.

\section{Limitations}

We acknowledge the preliminary nature of these findings, including its retrospective nature and limited sample size. As we included only patients with COVID-19 ARDS undergoing CTPA in this research, findings presented here cannot necessarily be extrapolated and have to be validated in a larger, and more heterogeneous COVID-19 cohort as well as a non-COVID cohort. Because pleural defects are nonspecific in B-mode, other examiners used contrast agent ultrasound (CEUS), with which the subpleural consolidations could be depicted even better. Trenker et al. found that, despite the lack of definite confirmation of PE on CT, peripheral subpleural consolidations with no or inhomogeneous enhancement on CEUS to be highly suggestive of embolic consolidations [21]. A follow-up study by the same team used histological examination of six cases, and pulmonary infarction was found in all of them [22]. This case series underlines that peripheral PEs are not necessarily discovered in the CTPA. When considering the recently published overview by Bérangère et al. [23] of the pathophysiological relationships of hemostasis in critically ill COVID-19 patients and the high risk of developing macro- and microthrombi due to hypercoagulability and endotheliopathy, it seems quite possible that there is not an overestimation of the PE-frequency in LUS, but instead an underestimation using CTPA as possible reasons for the false positive cases in the LUS. Unfortunately, contrast agent ultrasound exams were not part of our standardized LUS examination.

\section{Conclusions}

Large subpleural consolidations $\geq 1 \mathrm{~cm}$ detected in lung ultrasound were found frequently in COVID-19 ARDS patients with pulmonary embolism. In combination with a Wells score $>2$, PE could be predicted in COVID-19 ARDS with a sensitivity of $100 \%$ and a specificity of $80 \%$. This data might advocate a combination of LUS and the Wells score as screening tool for PE in COVID-19 ARDS.
A) typical thickening and irregularity of the pleura
B) small triangular subpleural consolidation $<1 \mathrm{~cm}$
C) non-typical polygonal subpleural consolidation
D) and E) PE-typical triangular subpleural consolidation $>1 \mathrm{~cm}$

F) PE-typical triangular subpleural consolid. $>1 \mathrm{~cm}$, with additional documentation after contrast agent 


\section{Abbreviations}

PE pulmonary embolism

ARDS acute respiratory distress syndrom

LUS lung ultrasound

CTPA computed tomography pulmonary angiography

ECMO veno-venous extracorporeal membrane oxygenation

COVID-19 Corona-Virus Disease 2019

SD standard deviation

BMI body mass index

PAP sys systolic pulmonary arterial pressure

SAPS2 simplified acute physiology core2

TISS therapeutic Intervention scoring system

ICU intensive care unit

DVT deep vein thrombosis

LMWH low molecular weight heparin

ROC receiver operating characteristic-curve

\section{Declarations}

\section{Ethics approval and consent to participate:}

The local ethics committee approved the study protocol (ethics committee of the University of Freiburg 234/20). Due to the retrospective design and observational nature of the study the need for informed consent was waived.

\section{Funding:}

The Baden-Wuerttemberg Ministry of Science, Research and Art and University of Freiburg funded the article processing charge.

Conflict of interests: 
The authors declare that they have no conflicts of interests.

\section{Availability of data and material}

The datasets of this study are available from the corresponding author on reasonable request.

\section{Permissions information:}

The authors declare that all illustrations and figures in the manuscript are entirely original and do not require reprint permission.

Consent for publication: We agree.

Code availability: not applicable.

Acknowledgements: not applicable.

Word count abstract: $\quad 271$

Word count manuscript: 3558 (manuscript only)

3974 (manuscript including tables and figures)

\section{Authors' contributions:}

VZ and CNL concepted and designed the study, performed data acquisition drafted the manuscript and created the artwork. A.S., X.B. and KMP, interpretation of the data, revised the manuscript.

CB and BS revised the manuscript. DS, TW and DD contributed in interpretation of the data and revising the manuscript. All authors read and approved the final manuscript.

\section{References}

1. CRICS TRIGGERSEP Group (Clinical Research in Intensive Care and Sepsis Trial Group for Global Evaluation and Research in Sepsis), Helms J, Tacquard C, et al (2020) High risk of thrombosis in patients with severe SARS-CoV-2 infection: a multicenter prospective cohort study. Intensive Care Med. https://doi.org/10.1007/s00134-020-06062-x

2. Llitjos J, Leclerc M, Chochois C, et al (2020) High incidence of venous thromboembolic events in anticoagulated severe COVID-19 patients. J Thromb Haemost jth.14869. https://doi.org/10.1111/jth.14869

3. Klok FA, Kruip MJHA, van der Meer NJM, et al (2020) Incidence of thrombotic complications in critically ill ICU patients with COVID-19. Thrombosis Research S0049384820301201. https://doi.org/10.1016/j.thromres.2020.04.013 
4. Tang N, Li D, Wang X, Sun Z (2020) Abnormal coagulation parameters are associated with poor prognosis in patients with novel coronavirus pneumonia. J Thromb Haemost 18:844-847. https://doi.org/10.1111/jth.14768

5. Chung M, Bernheim A, Mei X, et al (2020) CT Imaging Features of 2019 Novel Coronavirus (2019nCoV). Radiology 295:202-207. https://doi.org/10.1148/radiol.2020200230

6. Zhu J, Zhong Z, Li H, et al (2020) CT imaging features of 4121 patients with COVID-19: A metaanalysis. J Med Virol jmv.25910. https://doi.org/10.1002/jmv.25910

7. Zieleskiewicz L, Markarian T, Lopez A, et al (2020) Comparative study of lung ultrasound and chest computed tomography scan in the assessment of severity of confirmed COVID-19 pneumonia. Intensive Care Med 1-7. https://doi.org/10.1007/s00134-020-06186-0

8. Peng Q-Y, Wang X-T, Zhang L-N (2020) Findings of lung ultrasonography of novel corona virus pneumonia during the. Intensive Care Med 46:849-850. https://doi.org/10.1007/s00134-020-059966

9. Volpicelli G, Lamorte A, Villén T (2020) What's new in lung ultrasound during the COVID-19 pandemic. Intensive Care Med 46:1445-1448. https://doi.org/10.1007/s00134-020-06048-9

10. Manivel V, Lesnewski A, Shamim S, et al (2020) CLUE: COVID-19 lung ultrasound in emergency department. Emerg Med Australas 32:694-696. https://doi.org/10.1111/1742-6723.13546

11. Zotzmann V, Lang CN, Bamberg F, et al (2020) Are subpleural consolidations indicators for segmental pulmonary embolism in. Intensive Care Med 46:1109-1110. https://doi.org/10.1007/s00134-020-06044-z

12. Alhazzani W, Møller MH, Arabi YM, et al (2020) Surviving Sepsis Campaign: guidelines on the management of critically ill adults with Coronavirus Disease 2019 (COVID-19). Intensive Care Med 46:854-887. https://doi.org/10.1007/s00134-020-06022-5

13. Lichtenstein DA, Mezière GA (2008) Relevance of Lung Ultrasound in the Diagnosis of Acute Respiratory Failure*: The BLUE Protocol. CHEST 134:117-125. https://doi.org/10.1378/chest.072800

14. Mathis G, Blank W, Reissig A, et al (2005) Thoracic ultrasound for diagnosing pulmonary embolism: a prospective multicenter study of 352 patients. Chest 128:1531-1538. https://doi.org/10.1378/chest.128.3.1531

15. Moore AJE, Wachsmann J, Chamarthy MR, et al (2018) Imaging of acute pulmonary embolism: an update. Cardiovasc Diagn Ther 8:225-243. https://doi.org/10.21037/cdt.2017.12.01

16. Wells PS, Anderson DR, Rodger M, et al (2001) Excluding pulmonary embolism at the bedside without diagnostic imaging: management of patients with suspected pulmonary embolism presenting to the emergency department by using a simple clinical model and d-dimer. Ann Intern Med 135:98-107. https://doi.org/10.7326/0003-4819-135-2-200107170-00010

17. Nouvenne A, Zani MD, Milanese G, et al (2020) Lung Ultrasound in COVID-19 Pneumonia: Correlations with Chest CT on Hospital admission. Respiration 1-8. https://doi.org/10.1159/000509223 
18. Smargiassi A, Soldati G, Borghetti A, et al (2020) Lung ultrasonography for early management of patients with respiratory symptoms during COVID-19 pandemic. J Ultrasound 1-8. https://doi.org/10.1007/s40477-020-00501-7

19. Reißig A, Heyne JP, Kroegel C (2000) Diagnosis of pulmonary embolism employing transthoracic sonography: Comparison with helical computed tomography. Am J Respir Crit Care Med 161:A757

20. Reissig A, Heyne J-P, Kroegel C (2004) Ancillary lung parenchymal findings at spiral CT scanning in pulmonary embolism. Relationship to chest sonography. Eur J Radiol 49:250-257. https://doi.org/10.1016/S0720-048X(03)00141-4

21. Trenker C, Apitzsch JC, Pastor S, et al (2017) Detection of peripheral embolic consolidations using contrast-enhanced ultrasonography in patients with no evidence of pulmonary embolism on computed tomography: A pilot study. Journal of Clinical Ultrasound 45:575-579. https://doi.org/10.1002/jcu.22511

22. Trenker C, Dohse M, Ramaswamy A, et al (2019) Histological validation of pulmonary infarction detected with contrast-enhanced ultrasound in patients with negative computed tomography pulmonary angiogram: A case series. Journal of Clinical Ultrasound 47:461-465. https://doi.org/10.1002/jcu.22755

23. Joly BS, Siguret V, Veyradier A (2020) Understanding pathophysiology of hemostasis disorders in critically ill patients with COVID-19. Intensive Care Medicine 46:1603-1606. https://doi.org/10.1007/s00134-020-06088-1

\section{Figures}




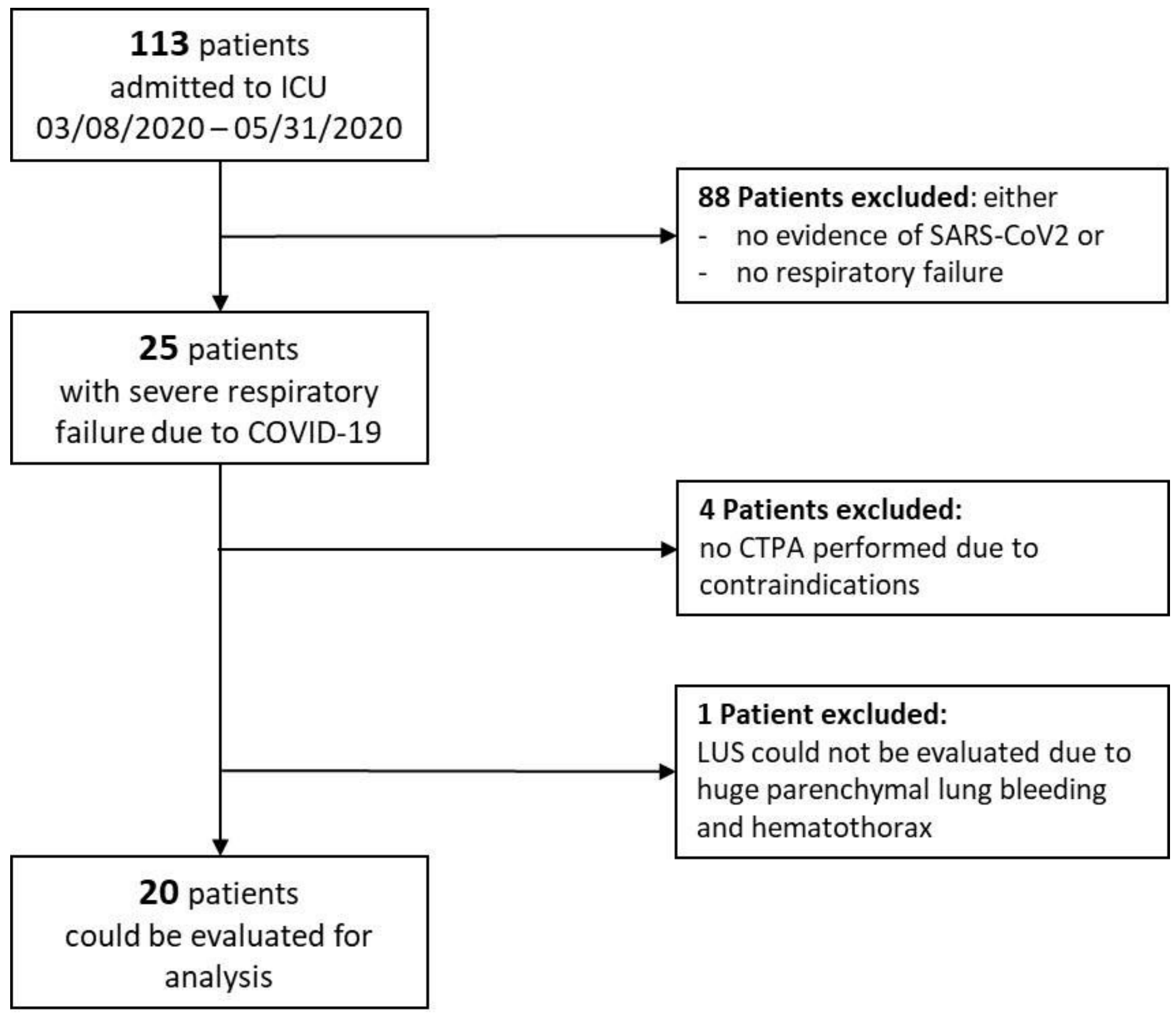

Figure 1

Flowchart patient selection. Abbreviations: CTPA computed tomography pulmonary angiography, COVID19 Corona-Virus Disease 2019, LUS lung ultrasound 


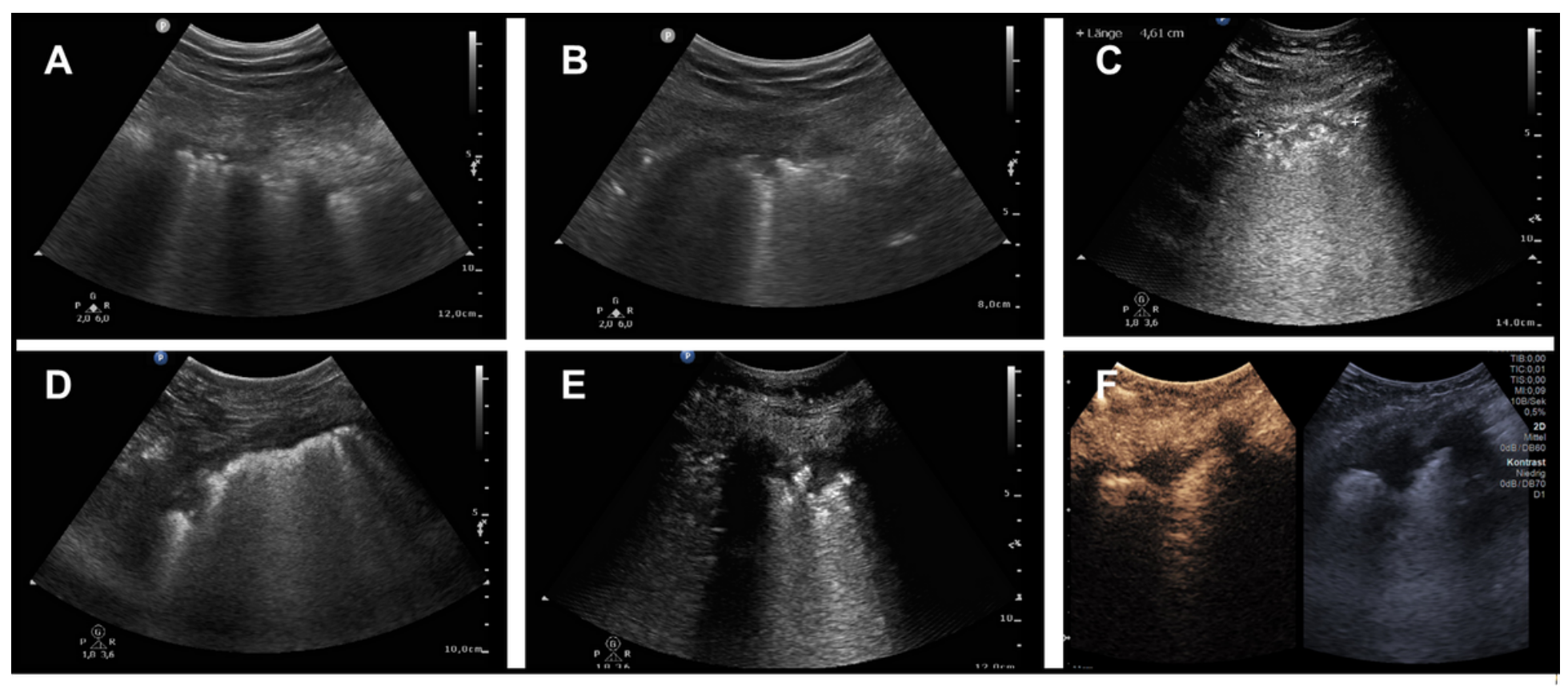

Figure 2

Sonographic image-examples of the pleural and subpleural changes in COVID-19 patients A) typical thickening and irregularity of the pleura B) small triangular subpleural consolidation $<1 \mathrm{~cm} \mathrm{C)} \mathrm{non-typical}$ polygonal subpleural consolidation $\mathrm{D}$ ) and E) PE-typical triangular subpleural consolidation $>1 \mathrm{~cm} \mathrm{~F}$ ) PEtypical triangular subpleural consolid. $>1 \mathrm{~cm}$, with additional documentation after contrast agent 
A Lung ultrasound

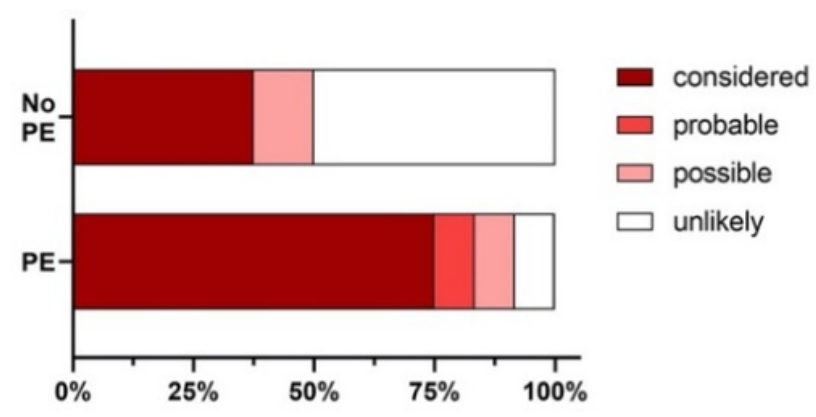

B Wells score

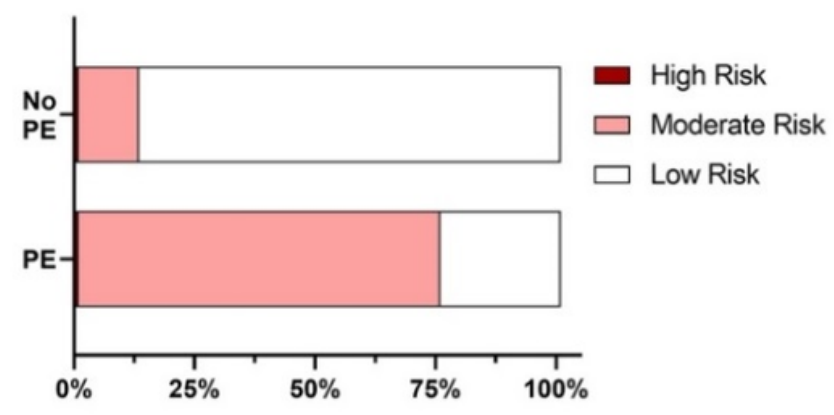

C Lung ultrasound plus Wells score

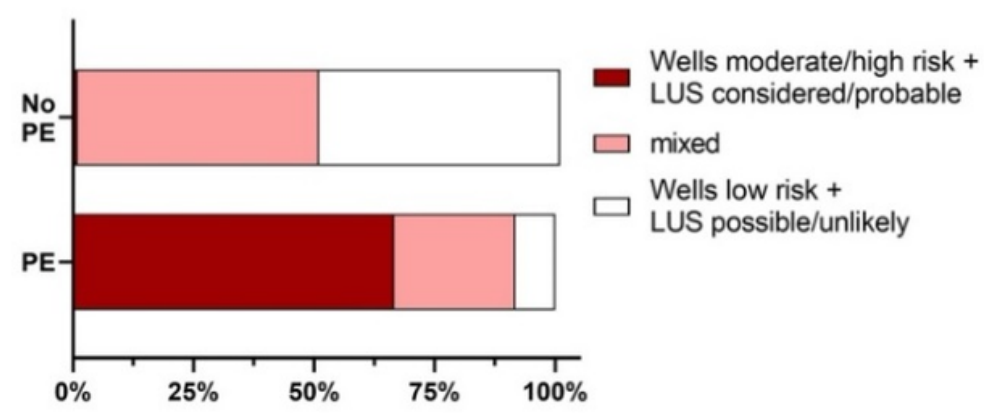

ROC for pulmonary embolism

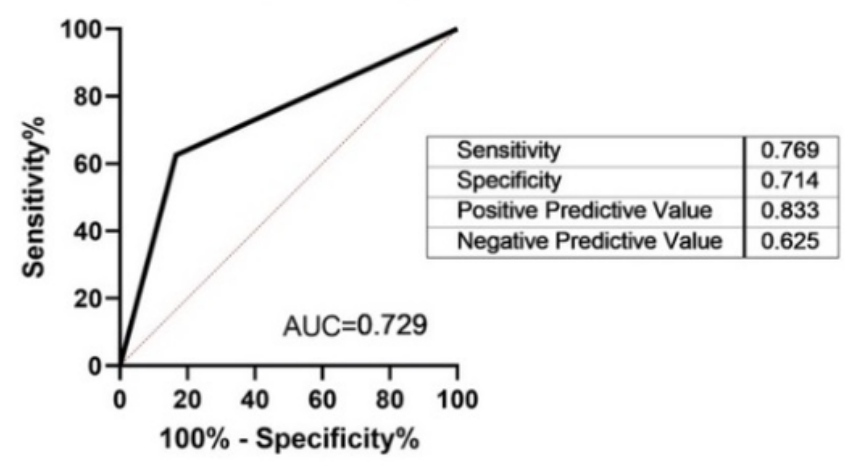

ROC for pulmonary embolism

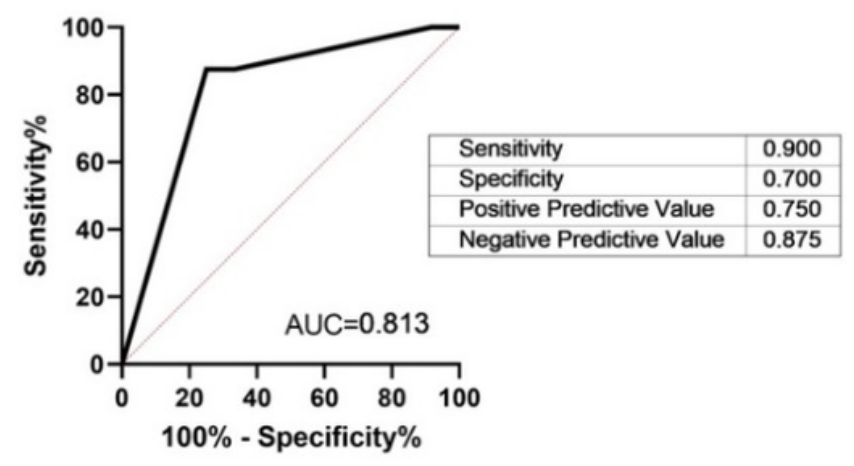

ROC for pulmonary embolism

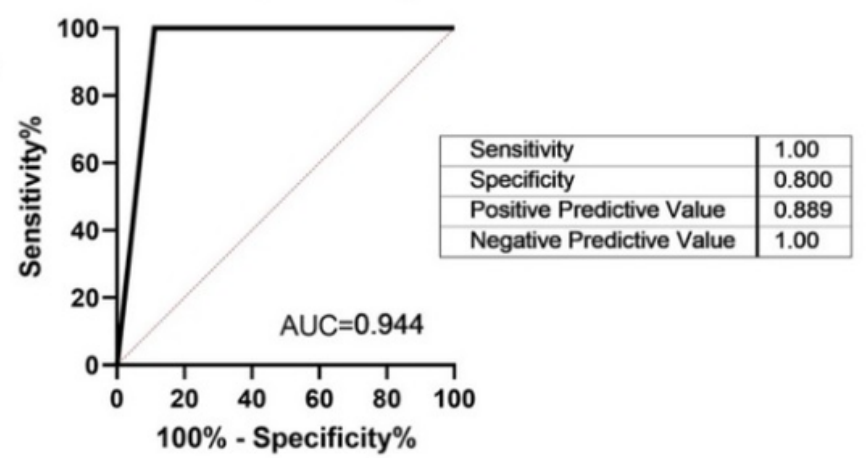

\section{Figure 3}

prediction of pulmonary artery embolism by lung ultrasound and wells score 